\title{
Ethical Considerations in Dental Epidemiological Studies
}

\author{
B. A. BURT
}

Program in Dental Public Health, School of Public Health, University of Michigan, Ann Arbor, Michigan 48109

J Dent Res 59(C):1289-1291, July 1980

Interpretation of the guidelines for ethical considerations in epidemiological studies requires defining a position of delicate balance. The goal must be to protect the rights, the health, and the dignity of study participants to the extent possible, while at the same time placing as few inhibitions as possible on the conduct of the research. The end result must therefore be a compromise, because these two requirements are inherently in conflict. The elements of this compromise are not static, for social philosophies change over time. What society considers the ideal balance between protection of the individual on the one hand and unconstrained research on the other will shift as these philosophies change. In addition, there can be cultural differences from one country to another at any one time. The steps which are currently regarded as necessary to provide acceptable protection of subjects in the United States may be seen as inadequate in some countries and excessive in others.

I believe that society is still in the process of defining this position of compromise. After some notorious abuses of the rights of participants in research studies conducted in previous years, ${ }^{1}$ the pendulum has now swung toward the establishment of more stringent conditions for protecting study participants. Some may question whether the pendulum has now swung far enough. A provocative editorial in Science suggested that complete disclosure of all aspects of a clinical trial should not be made to all participants, only to those who ask for it. ${ }^{2}$ The rationale was based on what the authors saw as the powerful negative aspects of the placebo effect.

Again, it must be stated that the balance sought is that between participants' rights and the smooth conduct of necessary research. If current guidelines really have overemphasized protection of participants at the expense of the conduct of necessary research, as this editorial in Science ${ }^{2}$ seemed to imply, then society ultimately will be the loser rather than the beneficiary.

Dr. Stamm has covered the area of ethical considerations in epidemiological dental studies with his usual thoroughness. $\mathrm{He}$ is to be commended for the way in which he has analyzed the ethical issues both in general and those applying to the hypothetical research protocol under consideration. I would like to reinforce some of his statements, and to bring up several issues upon which he did not touch, but which $I$ think are worthy of discussion. In the short time available I will confine my comments to general considerations rather than to the hypothetical research protocol, and I will emphasize the theme of the necessity to achieve an acceptable balance between the rights of participants and the conduct of necessary research.

\section{Relative risk.}

At the end of his paper, Dr. Stamm introduced the subject of what he called "relativism." "Relativism," in this context, means that although in dental research we can divide our experimentation into highrisk, moderate-risk, and low-risk categories, even our highest risk epidemiological studies can usually be classified as minimal-risk in the overall continuum of biomedical research.

Given this fact, I would agree with Dr. Stamm that the required pursuance, through an Institutional Review Board, of all the necessary clearance rituals for a dental epidemiological study is seen by some researchers as moralizing, and frequently as hardly necessary. The necessity of following current federal guidelines in explaining all the details of an epidemiological survey to prospective subjects, or their parents, produces a consent form that, even at a mini- 
mum, can be rather long. This could be seen as inhibiting conduct of the research without providing any additional protection to the subjects. Echoing the thinking in the Science editorial, ${ }^{2}$ it is possible that needless doubts could thus be planted in the minds of parents and children. There may be a tendency on the part of a prospective subject in a study to correlate the length of the consent form with the risks to which they think they may be exposed. In other words, no matter how clearly the absence of real risks in a dental epidemiological survey is explained to prospective participants, many may become sufficiently concerned to withhold consent simply because of the length of the consent form. It might make an interesting epidemiological study to see if, indeed, rates of participation in epidemiological studies are affected by the length of the consent form, but, of course, such a study would be unethical!

\section{Scientific validity.}

There seems to be acceptance, as Dr. Stamm pointed out, of the notion that the scientific validity of a study is indeed an ethical issue in itself. The rationale is that the time donated by study participants, and the inconvenience to which they are put, should at least be compensated for by the fact that the study in which they are taking part is scientifically valid and likely to produce some useful information. But the possibility of a classicial Catch-22 situation is then raised. To define it: Scientific validity of the study is an ethical requirement, and protection of participants is an ethical requirement. But if the operational requirements of subject protection lead to a degree of subject loss of sufficient magnitude to reduce the scientific validity of the study, which ethical issue has priority? To repeat the theme of this paper, we need to define a position of delicate balance between conflicting needs.

\section{Institutional review boards.}

Dr. Stamm suggested that Institutional Review Boards, which function primarily to examine the ethical issues of a research protocol, should also act as the first line of peer review. This suggestion is a logical extension of the idea that scientific validity per se is an ethical requirement. Dr. Stamm suggested that the peer review process would thus be tightened and that there would be a considerable saving in time, trouble and costs. While peer review of prospective research protocols, prior to their submission to a funding agency, is an excellent suggestion (one which many institutions already follow in one form or another). I would question whether an Institutional Review Board is an appropriate body for this task. Sometimes the Board must investigate technical merit in pursuing the finer points of ethics, as would have been the case in the hypothetical research protocol related to this discussion. But generally, in my opinion, the Institutional Review Boards have enough to do in ensuring that research protocols follow the current guidelines for protection of study participants. In addition, unless it so happened that a member of the Institutional Review Board was familiar with the area under study, it seems unlikely that the Board would be able to offer significant advice on structural details of the protocol.

\section{The needs of students.}

Finally, one other area we must consider is the need of students, particularly graduate students, who are in the process of learning how to conduct epidemiological studies. There is a partial analogy here with dental students who learn their techniques by treating consenting patients under supervision. There is a difference, however, between patients being treated by dental students and subjects being surveyed by a student epidemiologist, in that it is presumed that the dental patients receive a direct benefit to their health. It can be questioned whether subjects in an epidemiological survey are receiving a direct individual benefit.

In graduate training in dental public health, there are courses in which the graduate students, dentists studying towards their Masters degrees, go into the field to conduct their own surveys, again under direct supervision. The object of these courses is to give the students some practice in the conduct of field examinations, to let them experience the distinction between epidemiological and clinical examina- 
tions, to provide them with some practice in handling at least the more common dental indices, and to let them analyze data they have collected themselves. One population seen in the Michigan program involves the pupils at an elementary school, whose dentally-conscious principal likes to have the children in need of dental care identified. Some direct benefit is thus provided to the participants, while the survey meets the needs of our students. Another population, however, is a mentallyretarded group in a State hospital, which already has a dental treatment program. No direct individual benefit is provided to the participants in this case. Also, it cannot be said that new knowledge is accruing from these surveys. Their results are rarely, if ever, published, other than as term papers and student assignments.

\section{Summary.}

We therefore return to a point I made earlier, one which reiterates that made by Dr. Stamm, and that is the distinction between a benefit to the individual participant in an epidemiological survey and the benefit to society as a whole. I think we can generally agree with Dr. Stamm, and with other published guidelines, ${ }^{3}$ that epidemiological surveys usually provide little direct benefit to the participant (even the identification of a need for treatment might be questioned in some cases if the subject examined does not have access to dental care). Student surveys of the type 1 described can probably be justified on the basis of providing an eventual benefit to society in general, even though this benefit may not accrue until a future time. In my opinion, these student surveys thus meet the criteria for ethical acceptability.

The theme of this reaction paper has been the necessity to interpret ethical principles so that an acceptable balance can be achieved, protecting participating subjects without constraining necessary research. Therefore, I suggest that ethical guidelines for epidemiological surveys, probably more so than those in other areas of dental research involving human subjects, should be interpreted on what the benefits of these minimal risk procedures will be for society as a whole.

\section{REFERENCES}

1. HERSHEY, N. and MILLER, R.D.: Human Experimentation and the Law, Germantown, MD., Aspen Systems Corporation, 1976, pp. 1-11.

2. LOFTUS, E.F. and FRIES, J.F.: Informed Consent May Be Hazardous to Health, Science 204:11, 1979.

3. American Dental Association, American Association for Dental Research, and American Association of Dental Schools, Guidelines For the Use of Human Subjects in Dental Research, 1978. 